\title{
Angeborene und idiopathische Deformitäten: Immer ein Arthroserisiko?
}

\section{Congenital and Idiopathic Lower Limb Deformities: Always a Risk for Osteoarthritis?}

Autoren

Gregor Toporowski ${ }^{1}$, Robert Rödl ${ }^{1}$, Georg Gosheger ${ }^{2}$, Björn Vogt ${ }^{1}$

Institute

1 Abteilung für Kinderorthopädie, Deformitätenrekonstruktion und Fußchirurgie, Münster, Universitätsklinikum Münster

2 Klinik für Allgemeine Orthopädie und Tumororthopädie, Universitätsklinikum Münster, Münster

Schlüsselwörter

Präarthrotische Deformität, Kinderorthopädie, Sekundäre Arthrose

Key words

prevention, secondary osteoarthritis, paediatric

orthopaedics

Bibliografie

DOI https://doi.org/10.1055/a-1016-1332

Online-Publikation: 3.12.2019

Akt Rheumatol 2020; 45: 48-58

(c) Georg Thieme Verlag KG Stuttgart · New York ISSN 0341-051X

Korrespondenzadresse

Gregor Toporowski

Abteilung für Kinderorthopädie, Deformitäten-

rekonstruktion und Fußchirurgie

Universitätsklinikum Münster

Albert-Schweitzer-Campus 1

48149 Münster

Tel : + 49251 83-44180, Fax : + $4925183-47989$

gregor.toporowski@ukmuenster.de

\section{ZUSAMMENFASSUNC}

Arthrotische Gelenkveränderungen weisen eine Vielzahl an Ursachen auf. Ist eine eindeutige Zuordnung nicht möglich, spricht man von primären Arthrosen, hingegen kann die Entwicklung sekundärer Arthrosen auf konkrete Risikofaktoren zurückgeführt werden. Einen Großteil davon bilden angeborene und idiopathische Fehlstellungen, die auch als präarthrotische Deformitäten bezeichnet werden. Eine sekundäre Arthrose manifestiert sich in der Regel bereits in deutlich jüngerem
Lebensalter als ein primärer Gelenkverschleiß, gerade deshalb ist die rechtzeitige Diagnosestellung von entscheidender Bedeutung. Angeborene und idiopathische Deformitäten bleiben meist lange asymptomatisch, was die Diagnostik deutlich erschwert. In den letzten Jahren hat die Früherkennung häufiger Deformitäten jedoch stark an Bedeutung gewonnen, wie man am Beispiel der Sonografie zur Beurteilung der Hüftreifung im Rahmen der kindlichen Vorsorgeuntersuchung erkennen kann. Je nach Art der Deformität ist sowohl eine konservative, als auch eine operative Therapie möglich. Dabei spielt der richtige Zeitpunkt für den Therapiebeginn eine wichtige Rolle, denn sowohl ein Abwarten des Spontanverlaufs wie bei auffälligen frontalen Beinachsen, aber auch eine sofortige Behandlung wie bei der Hüftdysplasie können lege artis sein. Zu den häufigsten operativen Maßnahmen zählen die Wachstumslenkung und die Umstellungsosteotomie. Ziel der Therapie ist die Reduktion des Arthroserisikos auf das der Durchschnittsbevölkerung durch Wiederherstellung anatomisch und biomechanisch korrekter Stellungsverhältnisse. Folglich handelt es sich bei der Behandlung von präarthrotischen Deformitäten um präventive, gelenkerhaltende Maßnahmen, die in der Regel schonender und komplikationsärmer sind, als ein späterer endoprothetischer Gelenkersatz. Die Gefahr eines verspäteten Behandlungsbeginns ist die Notwendigkeit (aufwendigerer) operativer Interventionen.

\section{ABSTRACT}

Osteoarthritis (OA) is the most common joint disease and can be caused by numerous conditions. A large percentage of secondary OA cases is induced by congenital and idiopathic deformities of the lower limbs, which are often asymptomatic on clinical examination. As secondary OA usually occurs at a younger age than primary $\mathrm{OA}$, early diagnostic investigation and treatment are crucial. Depending on the type and severity of lower limb deformities, conservative and surgical treatment can be considered. Monitoring the natural course of a condition or starting early treatment can both be the correct treatment option. The purpose of treatment is to reduce the risk of OA to that of the average population by restoring the anatomically and biomechanically correct position. Consequently, the treatment of lower limb deformities consists in preventive, joint- 
preserving interventions and is usually less invasive and associated with fewer complications than endoprosthetic joint replacement performed at a later point in time. Depending on the residual growth, a delayed start of treatment may be associated with a (more complex) surgical intervention.

\section{Einleitung}

Zu den häufigsten Diagnosen in der orthopädischen Praxis zählen mit über $25 \%$ die angeborenen und erworbenen Deformitäten [1]. Unter ihnen macht der kongenitale Klumpfuß den größten Anteil aus, aber auch Erkrankungen wie die Hüftdysplasie als zweithäufigste angeborene Deformität oder bestimmte kniegelenksnahe Fehlstellungen müssen kinderorthopädisch therapiert werden, da sie eine präarthrotische Deformität darstellen [2].

Angesichts der zunehmenden Zahl älterer Menschen bei steigender Lebenserwartung ist zukünftig mit einer erhöhten Prävalenz degenerativer Gelenkerkrankungen zu rechnen. Dabei liegt die Lebenszeitprävalenz der Implantation einer Totalendoprothese bei Hüft- oder Kniegelenksarthrose aktuell bei knapp unter $25 \%$ [3]. Eine sekundäre Genese konnte bei Patienten mit Koxarthrose in 41,7\% und bei Patienten mit Gonarthrose in 33,4\% der Fälle nachgewiesen werden [4]. Gleichzeitig ist der endoprothetische Gelenkersatz bei sekundären Arthrosen aufgrund der zugrundeliegenden Fehlstellungen und Deformitäten technisch häufig anspruchsvoller und aufwendiger. Gerade deshalb geraten Diagnose und Therapie von präarthrotischen Deformitäten zuletzt vermehrt in den Fokus.

In diesem Artikel soll eine Übersicht über die Pathologie der häufigsten angeborenen und idiopathischen Deformitäten und die therapeutischen Möglichkeiten gegeben werden \ Tab. 1. Dabei soll insbesondere auf die Hüftdysplasie als häufigste kongenitale Deformität der Hüfte und das idiopathische Genu varum und valgum eingegangen werden.

\section{Ätiologie und Pathogenese}

\section{Konnatale Deformitäten}

1 bis $2 \%$ aller Neugeborenen weisen angeborene Fehlbildungen auf. Die Extremitätenfehlbildungen als eine heterogene Gruppe skelettaler Deformitäten stellen dabei nach den kardialen Fehlbildungen die am zweithäufigsten betroffene Lokalisation dar. Die Erstdiagnose wird vorrangig in den ersten drei kindlichen Vorsorgeuntersuchungen (U1-3) gestellt. Den größten Anteil bilden dabei Gliedmaßenfehlbildungen, die weniger auf genetische Ursachen, sondern vielmehr auf störende endogene oder exogene Einflüsse während der Embryonalentwicklung zurückzuführen sind $[5,6]$.

In dieser Zeit findet die Differenzierung der Extremitätenknospen zwischen der vierten und achten Schwangerschaftswoche statt. Dabei kommt es am Ende des Längenwachstums zu einer Veränderung der Lage: Die Armknospen rotieren nach lateral, die Beinknospen nach medial, sodass sich die Ellenbogenanlage schließlich dorsal und die Knieanlage ventral befinden. Diese Entwicklung ist der Grund für die physiologische Antetorsion des Femurs und die Bewegungsrichtung der Ellenbogen- und Kniegelenke.
Folglich ist in diesem Zeitabschnitt auch eine exogene Beeinträchtigung der Extemitätenreifung durch Infektionen, Hypoxie, Minderperfusion oder Toxine am ehesten möglich, weshalb er als vulnerable Phase bezeichnet wird. Genau genommen kann das klinische Bild einen Rückschluss auf den Zeitpunkt der Schädigung zulassen, da eine frühere Schädigung auch schwerere Deformitäten hervorruft $[6,7]$.

Eine Vielzahl konnataler Deformitäten ist zum Zeitpunkt der Geburt klinisch nicht sicher zu erkennen und manifestiert sich erst im weiteren Verlauf der körperlichen Entwicklung. Die genauen Ursachen von angeborenen Deformitäten sind zur Zeit nur in einem geringen Umfang geklärt und bleiben weiterhin Forschungsgegenstand [8-10].

\section{Die Hüftdysplasie}

Die zweithäufigste konnatale Deformität stellt die Hüftdysplasie mit oder ohne kongenitale Hüftgelenksluxation dar. Epidemiologisch lässt sich ein regionaler Unterschied feststellen, da eine vermehrte Häufung von Hüftreifungsstörungen in bestimmten Gebieten Deutschlands und Europas (sog. „Dysplasienester“) beschrieben wird.

Das Risiko einer Hüftreifungsstörung ist für Mädchen um das 6-fache höher als für Jungen [11, 12]. Interessanterweise beschreibt die Literatur eine signifikant erhöhte Erkrankungshäufigkeit der linken Seite. Dieses Phänomen wird damit begründet, dass der Fetus in der Embryonalentwicklung mit höherer Wahrscheinlichkeit mit dem Rücken zur linken Seite der Mutter liegt, womit das linke Bein vermehrt von ventral in Adduktion gedrückt wird [13].

Neben dem weiblichen Geschlecht gelten vornehmlich die Beckenendlage und die familiäre Belastung als weitere Risikofaktoren.

\section{Idiopathische und sekundäre Deformitäten}

Die am häufigsten festgestellten präarthrotischen Deformitäten sind nicht angeboren, sondern entwickeln sich aus einer zunächst anatomisch und biomechanisch korrekten Stellung heraus. Hierzu zählen insbesondere das Genu varum und valgum sowie das femoroazetabuläre Impingement (FAI). Bei der Entstehung dieser Deformitäten kann einerseits ein gestörter Mineralhaushalt, wie er bspw. bei Phosphatdiabetes auftritt, andererseits aber auch eine dauerhafte biomechanische Fehlbelastung, wie sie unter anderem bei Patienten mit Achondroplasie zu finden ist, ursächlich sein. Am häufigsten bleibt die Ätiologie jedoch unklar.

Die Ursache für die Entstehung eines FAl scheint in einer erhöhten Belastung der Wachstumsfuge des proximalen Femurs während eines Wachstumsschubes begründet zu sein. Häufig kann bei Jugendlichen, die im präpubertären Alter sportlich sehr aktiv waren, eine sog. Cam-Deformität mit reduzierter Schenkelhalstaillierung nachgewiesen werden. Insbesondere im Zusammenhang mit bestimmten Bindegewebserkrankungen findet sich umgekehrt eine vermehrte azetabuläre Überdachung des Hüftkopfes, welche 
> Tab. 1 Beispiele präarthrotischer Deformitäten, sortiert nach Prävalenz.

\begin{tabular}{|c|c|c|c|}
\hline Erkrankung & Prävalenz & Auswirkung & Therapie \\
\hline Femuroacetabuläres Impingement & $14 \%$ & Koxarthrose & Arthroskopie, Umstellungsosteotomie \\
\hline Genu varum oder valgum & $12 \%$ & Gonarthrose & Wachstumslenkung, Umstellungsosteotomie \\
\hline Hüftdysplasie & $4 \%$ & Koxarthrose & Orthesenbehandlung, Periazetabuläre Umstellung \\
\hline Epiphyseolysis capitis femoris & $0,1 \%$ & Koxarthrose & Offene Reposition, Verschraubung \\
\hline $\begin{array}{l}\text { Longitudinaler Reduktionsdefekt der } \\
\text { unteren Extremität }\end{array}$ & $1: 2000$ & Koxarthrose Gonarthrose & $\begin{array}{l}\text { Hilfsmittelversorgung, Operative Rekonstruktion, } \\
\text { Kallusdistraktion }\end{array}$ \\
\hline M. Perthes & $1: 3000$ & Koxarthrose & Physiotherapie, Entlastung, Umstellungsosteotomie \\
\hline Coxa vara congenita & $1: 25000$ & Koxarthrose & Umstellungsosteotomie \\
\hline Coxa retrotorta & $1: 50000$ & Koxarthrose & Umstellungsosteotomie \\
\hline
\end{tabular}

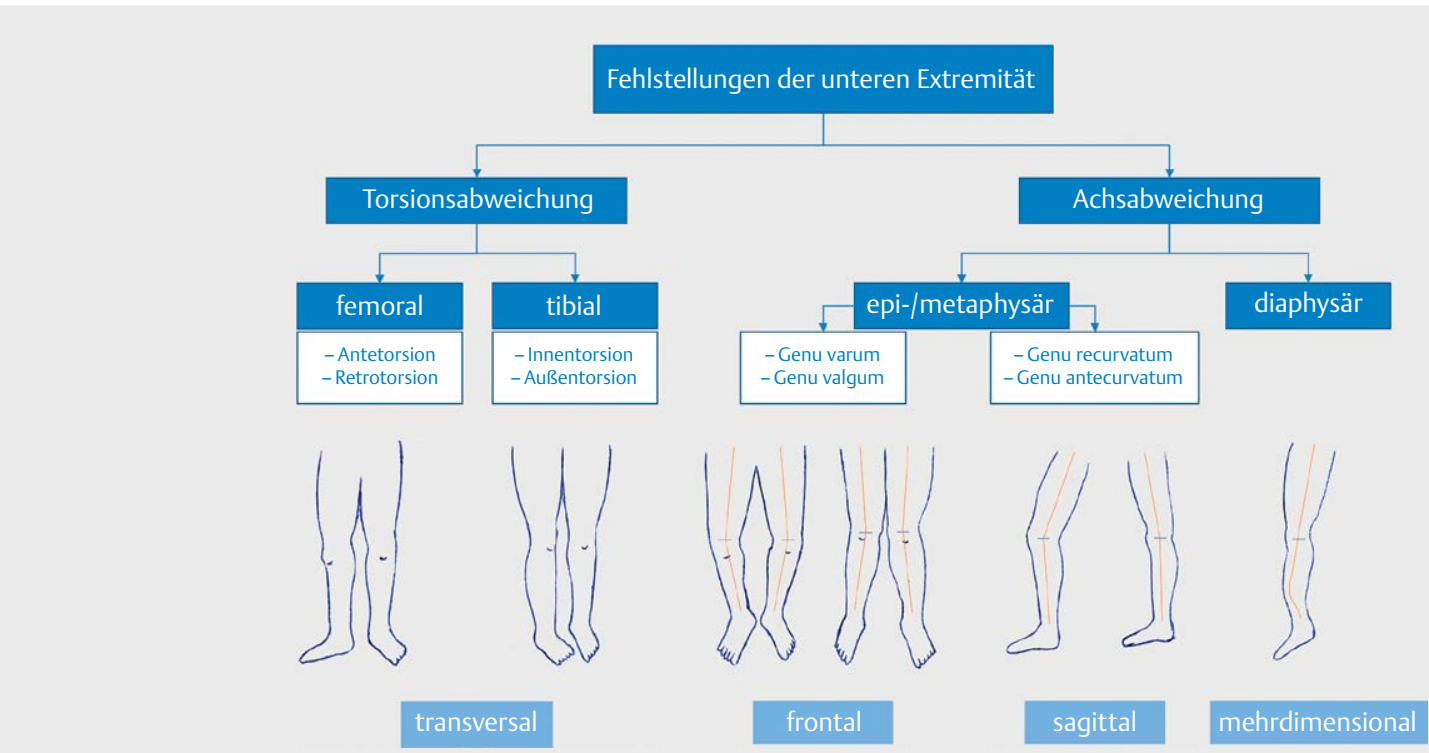

- Abb. 1 Fehlstellungen der unteren Extremität in der Frontal-, Sagittal- und Transversalebene

zu Bewegungseinschränkungen führen kann. Diese sog. Pincer-Deformitäten können durch azetabuläre Fehlstellungen (Antetorsion bzw. Retroversion) oder Vertiefungen (Protrusio acetabuli bzw. Coxa profunda) entstehen. Im klinischen Alltag werden selten isolierte FAI-Formen, sondern vielmehr eine Kombination aus Camund Pincer-Deformität vorgefunden. In den meisten Fällen kann hier jedoch keine genaue Ursache bestimmt werden [14].

\section{Entwicklung von Deformitäten der unteren Extremität}

Grundsätzlich kann eine fehlerhafte biomechanische Belastung eines Gelenks im Verlauf zu einer Arthrose führen. In \ Abb. 1 sind mögliche fehlerhafte Entwicklungen der Achs- und Torsionsverhältnisse der unteren Extremität aufgelistet.

Nicht jede auffällige Beinstellung muss unbedingt als pathologisch angesehen werden. So lässt sich in der Frontalebene postna- tal ein Crus varum vorfinden. Nach der Vertikalisierung führt die asymmetrische Belastung der kniegelenksnahen Wachstumsfugen zu einer entgegengesetzten Entwicklung der Beinachse und somit zum Genu valgum mit einem Maximum im Alter von 3 bis 4 Jahren. Die wiederum veränderte Gewichtsbelastung der Wachstumsfugen bedingt bis zum 10. Lebensjahr die Entstehung einer neutralen frontalen Achse ( $\triangleright$ Abb. 2) $[7,15,16]$.

Schaut man sich die Entwicklung der unteren Extremität in der Transversalebene an, so misst der Winkel zwischen Schenkelhals und Femurkondyle postnatal physiologischerweise ca. $30^{\circ}$. Nach der Vertikalisierung reduziert sich dieser Antetorsionswinkel stetig über die Jahre, beschleunigt in 2 sog. Akzelerationsphasen, auf ca. $10-15^{\circ}$ bei Wachstumsabschluss ( $\triangleright$ Abb. 3 ). Die Tibia hingegen ist bei Geburt in der Regel nicht torquiert, dreht sich jedoch bis zum Alter von 4-6 Jahren belastungsbedingt auf ca. $20-25^{\circ}$ Außentorsion [17]. 

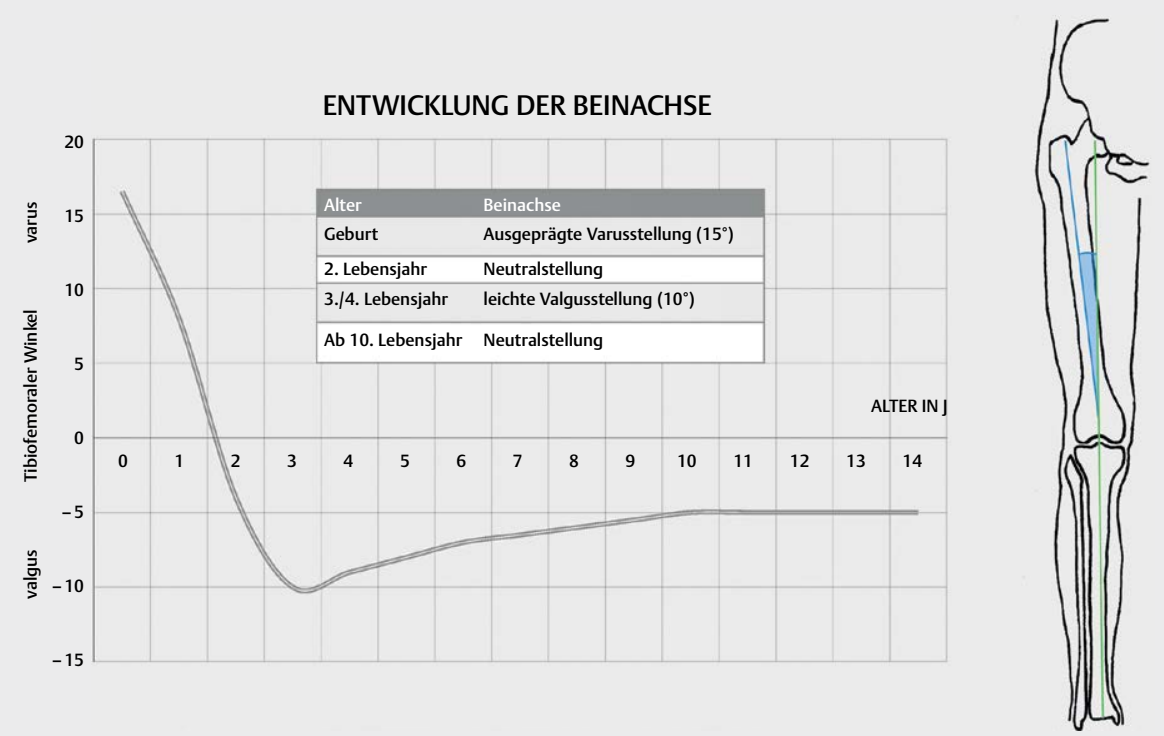

Abb. 2 Entwicklung der frontalen Beinachse nach Salenius u. Vankka [16].

\section{ENTWICKLUNG DER FEMORALEN ANTETORSION}

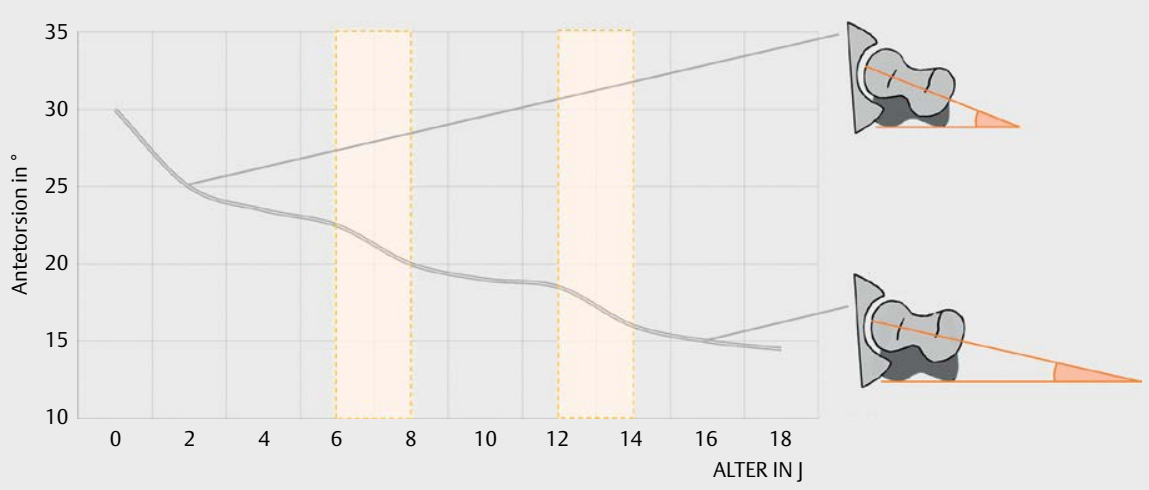

- Abb. 3 Entwicklung der femoralen Antetorsion [18-19].

In all diesen Phasen können folglich Entwicklungsstörungen auftreten, die in Fehlstellungen resultieren. Jedoch stellt nur ein geringer Anteil davon - vornehmlich die ausgeprägte frontale Fehlstellung - eine präarthrotische Deformität dar.

\section{Biomechanische und zelluläre Grundlagen}

Der Begriff der „präarthrotischen Deformität“ wurde bereits 1943 von M. Hackenbroch verwendet und als Ursache einer „Arthrosis deformans“ beschrieben. Die in der Regel stark belasteten, zueinander kongruenten Gelenkflächen werden aufgrund vorherrschender Deformität verkleinert und in dessen Folge inkongruent. Entsprechend erhöht sich der Druck auf die verbliebene Fläche, verstärkt die Inkongruenz und führt über Umstrukturierung der Knorpelmatrix zur Degeneration [20].
Am Beispiel des Hüftgelenks bestimmt in erster Linie die Überdachung des Hüftkopfes - das sogenannte „Containment“ - die Größe der Gelenkfläche und somit die Druckbelastung. Ist die Hüftpfanne zu klein und zu steil, spricht man von der Hüftgelenksdysplasie. Durch die Verkleinerung der Artikulationsfläche erhöht sich der punktuelle Druck, was zu vorzeitigem Knorpelschaden bis hin zur knöchernen Deformation führt ( $>$ Abb. 4a). Ein Hypercontainment hingegen, wie es bei der Coxa profunda bzw. der Protrusio acetabuli vorgefunden wird, verringert das Ausmaß der Beweglichkeit im Hüftgelenk. Dabei wird durch die Schädigung des Labrum acetabuli und des angrenzenden Knorpels durch ständigen femoroazetabulären Kontakt die frühe Degeneration erzeugt ( $\triangleright \mathbf{A b b}$. 4b) [21].

Die Kraftübertragungslinie vom Hüftkopfmittelpunkt bis zur Mitte des Sprunggelenks wird als Mikulicz-Linie bezeichnet. Sie verläuft in der Regel durch das Zentrum des Kniegelenks, was zur 


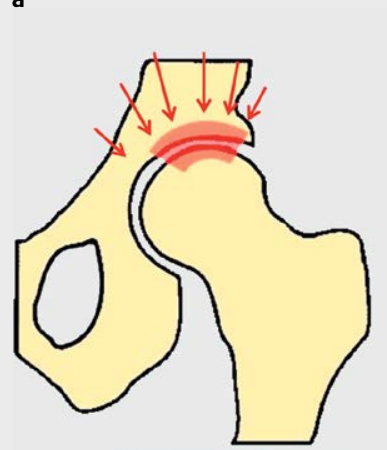

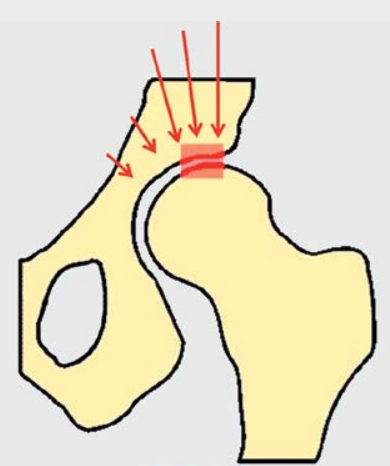

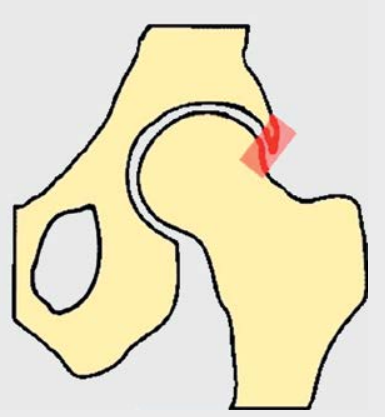

Abb. 4 a Verringerung der Gelenkfläche führt zur Erhöhung und Verschiebung des punktuellen Drucks (Pfeile) auf einen Teil des Hüftgelenks,

b Vergrößerung der Gelenkfläche führt zum Pincer-Impingement.

gleichmäßigen Belastung des medialen und lateralen KniegelenkKompartimentes im seitengleichbelasteten Zweibeinstand führt. Kommt es zu einem Genu varum, verlagert sich der Schnittpunkt der Mikulicz-Linie mit der Kniegelenksachse nach medial, weshalb sich die Belastung medial erhöht. Entsprechend andersherum verhält sich die Biomechanik bei einem Genu valgum ( $\bullet$ Abb. 5a).

Im Alltag wird das Kniegelenk jedoch den größten Belastungsspitzen im Gehen oder Laufen und somit im einbeinigen Stand ausgesetzt. Folglich ergibt sich hierbei eine deutlich höhere Belastung im medialen Kniegelenk ( $\boldsymbol{A} \mathbf{A b} \mathbf{b}$. $\mathbf{5 b}$ ). Diese wird bei intakter muskulärer Konstitution durch den Tractus iliotibialis vermindert, woraus die Gesamtbelastung resultiert. Eine unzureichende Funktion des Tractus iliotibialis wird in der Literatur als Ursache für die deutlich erhöhte Anzahl an medialer Gonarthrose beschrieben [22, 23].

Zu den wesentlichen makroskopischen Veränderungen eines arthrotischen Gelenks zählt der Knorpelschaden. Biologisch ist hyaliner Gelenkknorpel sehr zellarm und avaskulär, weshalb eine Regeneration bei entstandenem Schaden nur sehr eingeschränkt möglich ist. Liegt eine punktuell vermehrte Belastung eines Gelenkknorpelabschnitts infolge einer biomechanischen Fehlstellung vor, wird in Folge dieser Knorpelanteil verstärkt destruiert, weshalb eine frühere Arthrosebildung möglich ist [24].

\section{Diagnostik}

Eine angeborene oder idiopathische Deformität der unteren Extremität sollte möglichst früh diagnostiziert werden, um den entsprechenden Therapiebeginn nicht zu verpassen. Dieser liegt bei der Hüftdysplasie aufgrund guter konservativer Therapiemöglichkeiten im Säuglingsalter deutlich früher als bei kniegelenksnahen Fehlstellungen. Abhängig vom Alter der Diagnosestellung muss auf den bereits erwähnten physiologischen Entwicklungsverlauf der unteren Extremität geachtet werden, denn nicht jede fehlerhaft anmutende Konstitution ist auch pathologisch.

Nachfolgend sind die diagnostischen Strategien bei den häufigsten präarthrotischen Deformitäten beschrieben, wobei aus didaktischen Gründen eine chronologische Reihenfolge sortiert nach typischem Diagnosealter eingehalten wurde ( $\bullet$ Tab. 2).

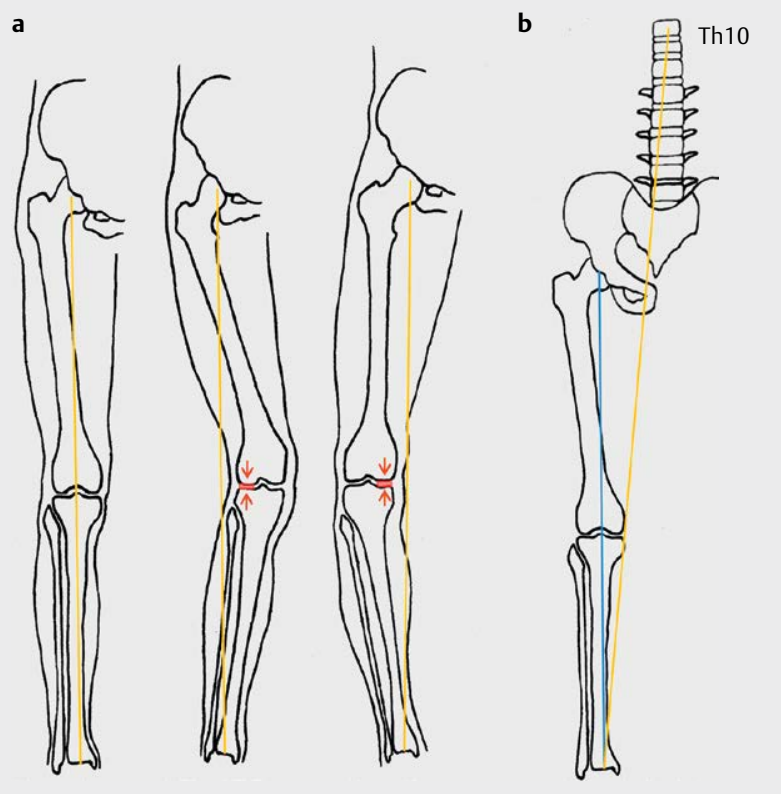

Abb. 5 a Neutrale, valgische und varische Kniegelenksachse. Die rot markierten Areale sind bei veränderter Biomechanik verstärkt belastet, $\mathbf{b}$ Die Belastungsachse (gelb) verschiebt sich im Einbeinstand nach medial.

\section{Säuglingsalter}

\section{Hüftdysplasie}

Wie bereits beschrieben, stellt die Hüftdysplasie die zweithäufigste konnatale Deformität dar, weshalb ihre frühzeitige Diagnose von besonderer Bedeutung ist. Entsprechend findet das obligate Screening mittels Hüftsonographie spätestens in der U3 zwischen der 4. und 6. Lebenswoche statt. Bei Vorliegen eines oder mehrerer der voranstehend aufgeführten Risikofaktoren für eine Hüftdysplasie, wird in der Regel bei der U2 in den ersten Tagen nach Geburt eine sonographische Untersuchung der Hüften durchgeführt [25]. 
- Tab. 2 Typisches Diagnosealter von Deformitäten der unteren Extremität, chronologisch sortiert.

\begin{tabular}{|l|l|}
\hline Alter in J & Erkrankung \\
\hline $0-2$ & - Hüftdysplasie \\
\hline & " Fußdeformitäten \\
& " Reduktionsdefekt \\
& Beinlängendifferenz \\
\hline $2-16$ & - M. Perthes \\
\hline & " Epiphyseolysis capitis femoris \\
& - Achs-/Torsionsabweichungen \\
\hline & - Femoroacetabuläres Impingement \\
\hline
\end{tabular}

Die klinische Untersuchung des Hüftgelenks im Säuglingsalter ist vor allem für die Feststellung einer Hüftgelenksluxation von Bedeutung. Ein stabiles, ausschließlich dysplastisches Hüftgelenk zeigt sich klinischen unauffällig und entgeht somit der körperlichen Untersuchung. Entsprechend ist hierbei die strukturierte sonografischen Untersuchung der diagnostische Goldstandard.

Folgende klinische Zeichen für eine (unilaterale) Hüftgelenks (sub)luxation können vorliegen > Abb. 6a-c:

- Faltenasymmetrie (a)

- Abduktionshemmung(b)

- Positives Galeazzi-Zeichen (c)

Die Faltenasymmetrie tritt gelegentlich auch bei gesunden Säuglingen auf, entsprechend gilt es als unspezifisches Zeichen.

Normalerweise weisen Neugeborene aufgrund einer Kontraktur der ischiokruralen Muskulatur durch mehrmonatige Flexionshaltung der Kniegelenke im Mutterleib eine leichte Kniebeugekontraktur auf. Bei Hüftluxation werden Ursprung und Ansatz der Hamstrings einander angenähert. Deshalb ist bei luxiertem Hüftgelenk dann meist eine volle Kniestreckung möglich (positives Ludloff-Zeichen). Auch dieses klinische Zeichen wird meist bei unilateraler Luxation als Seitenasymmetrie festgestellt. Die Barlow- und Ortolani-Zeichen, bei denen sich das Hüftgelenk luxierbar und reponierbar zeigt, werden aufgrund der potenziellen Labrumschädigung nicht mehr durchgeführt.

Da die körperliche Untersuchung der Säuglingshüfte größtenteils auf dem Seitenvergleich beruht, ist bei beidseitiger Hüftgelenksluxation eine klinische Beurteilung deutlich erschwert.

Das Hüftgelenk besteht zum Zeitpunkt der Geburt zum großen Anteil aus Knorpelgewebe, weshalb eine Beurteilung im Röntgen kaum bis gar nicht möglich ist. Entsprechend ist die Ultraschalluntersuchung nach Graf in Europa der Goldstandard zur Darstellung des Hüftgelenks in diesem Alter. Hierbei werden die Morphologie des knöchernen und knorpeligen Erkers sowie die knöcherne ( $\alpha$-Winkel) und knoprelige Überdachung ( $\beta$-Winkel) des Hüftkopfs beurteilt und in die Typen I-IV eingeteilt. Typ I nach Graf entspricht einer regelhaft ausgereiften Hüfte, die im weiteren Verlauf keine Tendenz zur Verschlechterung zeigt. Typ 2 ist durch eine zentrierte Position des Hüftkopfs unter dem Pfannendach gekennzeichnet, wobei das Pfannendach eine Verzögerung der Verknöcherung aufweist. Typ III beschreibt eine mangelhafte knöcherne Überdachung mit einem abgeflachten Erker, einem nach kranial dislozierten knorpeligen Pfannenrand und gleichzeitig eine Dezentrierung des Hüftkopfs. Beim Typ IV handelt es sich um ein vollständig luxiertes Hüftgelenk [26].

Ist die sonografische Darstellung der Hüfte nicht möglich, kann ab dem 3. Lebensmonat eine Röntgenuntersuchung mittels Becken a.p. durchgeführt werden. Diese Röntgenuntersuchung wird in der AWMF-Leitlinie jedoch erst ab dem 9. Monat empfohlen [25].

\section{Laufbeginn}

Eine Beinlängendifferenz oder gar ein longitudinaler Reduktionsdefekt der unteren Extremität kann je nach Ausprägung bereits im ersten Lebensjahr auffallen.

Die klinische Untersuchung gibt darüber Aufschluss, wie stark das Bewegungsausmaß der Extremität eingeschränkt und ob eine konservative Unterstützung zum Erlernen des Stehens und Gehens frühzeitig notwendig ist. Zum anderen kann nach Berechnung der zu erwartenden Beinlängendifferenz, z. B. nach der Multiplier Methode, mit den Eltern diskutiert werden, ob eine operative und / oder konservative Therapie im weiteren Verlauf sinnvoll ist [27].

In der radiologischen Untersuchung in Becken a.p.- und Lauensteindarstellung kann einerseits die Verlaufskontrolle von therapierten Hüftdysplasien erfolgen, andererseits die Stellung des proximalen Femurs beurteilt werden. Eindimensionale Fehlstellungen des Schenkelhalses sind dabei selten [28]. In diesem Zusammenhang sind bspw. die Coxa vara et retrotorta zu erwähnen, aber auch schwerwiegende Fehlbildungen wie ein femoraler Felddefekt kann in seiner Ausprägung beurteilt und weitere Therapieschritte geplant werden.

\section{Kleinkind-/Jugendalter}

Achsfehlstellung

Eine angeborene Achsfehlstellung der unteren Extremität ist sehr selten, entsprechend ist der häufigste Diagnosezeitraum erst nach Laufbeginn. Dabei ist auf die bereits erwähnte physiologische Entwicklung der Beinachsen im Verlauf zu achten ( $\mathbf{A} \mathbf{b b}$. 2). Die häufigsten Achsendeviationen finden sich in der Frontalebene und haben aufgrund der Schanierfunktion des Kniegelenks die höchste klinische Bedeutung und die geringsten mechanischen Kompensationsmöglichkeiten [29].

Nur ein geringer Anteil der Patienten mit Achsfehlstellungen in der Frontalebene ist primär symptomatisch und weist Schmerzen im betroffenen medialen oder lateralen Kniegelenksanteil oder stellungsbedingte Funktionsdefizite auf. Klinisch wird zunächst die Beinachse im Stand aus frontaler und sagittaler Richtung betrachtet. Bei einem Genu varum liegt ein erhöhter Interkondylarabstand vor, ein Genu valgum ist durch einen erhöhten Intermalleolarabstand gekennzeichnet. Bei adipösen Kindern ist aufgrund des vermehrten Oberschenkelumfangs gelegentlich ein erhöhter Intermalleolarabstand feststellbar, obwohl kein Genu valgum vorliegt. Hier kann die radiologische Korrelation Aufschluss geben.

Das wichtigste radiologische Diagnoseinstrument bei frontalen Achsfehlstellungen ist die Ganzbeinstandaufnahme. Hier ist es besonders wichtig seitens der durchführenden radiologischen Abteilung darauf zu achten, dass die Kniegelenke durchgestreckt und 
die Kniescheiben exakt nach vorne ausgerichtet sind. Liegt ein Genu varum vor, befindet sich das Kniegelenkszentrum lateral der Mikulicz-Linie, bei einem Genu valgum entsprechend medial davon [30] Abb. 5 a.

Eine Unterscheidung zwischen femoral oder tibial bedingter Fehlstellung kann anhand der Messung der kniegelenksnahen Winkel zwischen Kniebasis und den mechanischen Achsen des Femurs bzw. der Tibia getroffen werden. Der mechanische laterale distale Femurwinkel (mLDFW) und der mediale proximale Tibiawinkel (MPTW) haben jeweils einen Normwert von $87 \pm 3^{\circ}$ [22].

Wird beim Patienten ein retardiertes oder akzeleriertes Skelettalter im Gegensatz zum chronologischen Alter vermutet, kann eine Skelettalterbestimmung beispielsweise anhand von d.p.-Aufnahmen der linken Hand nach Greulich und Pyle [31] Aufschluss über noch vorhandenes Restwachstum und Korrekturpotenzial und somit den optimalen Interventionszeitpunkt geben.

Besteht bei einer präarthrotischen Deformität im Jugend- oder frühen Erwachsenenalter die Indikation zur operativen Korrektur, kann in komplexen Fällen vorab untersucht werden, in welchem Ausmaß eine Knorpelschädigung vorliegt. In Erweiterung der nativradiologischen Routinediagnostik kann mittels MRT-Untersuchung des betroffenen Gelenks die Morphologie und Höhe der hyalinen Knorpelschicht ermittelt und somit abgewogen werden, ob eine gelenkerhaltende operative Therapie bei noch ausreichend gutem Knorpelzustand sinnvoll ist [32].

In den letzten Jahren ist die Diagnostik und Einstufung einer vorliegenden Knorpelpathologie deutlich verbessert worden. Zur longitudinalen Verlaufskontrolle, insbesondere von rheumatologischen Gelenkerkrankungen, etabliert sich zunehmend das biochemische MRT, bei dem bspw. der Glycosamingehalt des Knorpels und sein möglicher Verlust quantifiziert werden [33]. Auch in der Beurteilung posttraumatischer präarthrotischer Deformitäten findet diese diagnostische Maßnahme zunehmend Verwendung [34], sodass diese Technik zukünftig möglicherweise ebenfalls zur Einschätzung des Knorpelstatus bei kongenitalen Gelenkdeformitäten eine Rolle spielen kann.

\section{Therapie}

Abhängig von der Art und Lokalisation der präarthrotischen Deformität ist sowohl das Abwarten des Spontanverlaufs als auch sofortiger Therapiebeginn möglich. Grundsätzlich sollten konservative Maßnahmen eher früh durchgeführt werden, um das körpereigene Korrekturpotenzial durch Optimierung der Biomechanik zu nutzen. Hingegen sollten wachstumslenkende operative Eingriffe zeitlich so geplant werden, dass einerseits noch ausreichend Potenzial zur Korrektur der Fehlstellung vorliegt, andererseits nach erreichter Korrektur jedoch nur noch möglichst wenig Restwachstum vorhanden ist, um ein Wiederauftreten der Deformität zu vermeiden.

\section{Hüftdysplasie}

Die Behandlung einer vorliegenden Hüftreifungsstörung sollte so früh wie möglich begonnen werden, da die Wachstumsgeschwindigkeit und Ossifikation des Pfannendachs insbesondere in den ersten vier Lebenswochen sehr hoch ist und im Verlauf abnimmt. Ab der 16. Lebenswoche ergibt sich hier kaum noch eine strukturelle Veränderung [35].

Kann in der klinischen Untersuchung und in der Sonografie eine stabile Hüftgelenkssituation ohne Luxations- oder Subluxationstendenz ausgemacht werden, ist eine Behandlung mittels Spreizschiene (z. B. Tübinger Beugeorthese) für mindestens 6 Wochen sinnvoll. Hierbei wird die Hüfte um etwa $110^{\circ}$ flektiert und $50^{\circ}$ abduziert, um die Sitzhockstellung zu erreichen, die der Säugling bereits als Fetus zur Hüftreifung innehatte („human position“ nach Salter). Ziel dieser Therapie ist die Retention des Hüftkopfs zur Induktion der Nachreifung der Pfanne sowie die Vermeidung von (Sub-)luxationsstellung. Unter 6-wöchigen sonografischen Kontrollen sollte hierdurch schließlich ein $\alpha$-Winkel von deutlich über $60^{\circ}$ erreicht werden, bevor eine Abschulung der Schiene stattfinden sollte.

Im Falle einer (Sub-)luxation (Typ III und IV) kann in den meisten Fällen eine Reposition durch konservative Therapiemaßnahmen mithilfe von Orthesen (z. B. Pavlik-Bandage) erreicht werden. Bei allen Orthesenanlagen ist darauf zu achten, dass das Hüftgelenk in der sog. „Safe zone“ nach Ramsey eingestellt wird, um das Risiko einer Hüftkopfnekrose zu verringern. Ist eine sichere Retention des Hüftgelenks mit Orthese / Bandage nicht möglich, kann die Anlage eines Becken-Bein-Gipses (z. B. nach Fettweis) in Sedierung in Sitzhockstellung unter leichtem Druck am proximalen Femur nach ventral erfolgen. Die Gipsanlage erfolgt meist für ca. 3-6 Wochen, hiernach ist in der Regel die Anlage der Spreizbandage bis zur vollständigen Ausheilung möglich [36].

Bei Vorliegen eines Repositionshindernisses und ausbleibender Repositionsmöglichkeit kann im Alter von 3 Monaten eine Arthrographie und ein erneuter geschlossener Repositionsversuch durchgeführt werden. Gelingt dieser nicht, ist der Wechsel auf eine offene chirurgische Reposition notwendig. Im Anschluss erfolgen ebenfalls die Anlage eines Fettweis-Gipses sowie die anschließende Spreizschienenbehandlung [36]. Die offene Reposition kann ebenfalls mit einer Azetabuloplastik kombiniert werden, wodurch die Nachreifungsphase enfällt.

Wird mit ca. 1,5 Jahren in der radiologischen Bildgebung mittels Becken a.p. eine unzureichende Hüftgelenksüberdachung festgestellt, wird eine Azetabuloplastik empfohlen ( $\mathbf{A}$ Abb. 7). Dieser Eingriff kann spätestens bis zum 5.-6. Lebensjahr durchgeführt werden, so lange die Y-Fuge des Beckens als Drehpunkt der Korrektur noch nicht partiell verknöchert ist.

Wird die Diagnose der Hüftdysplasie erst nach Verknöcherung der Y-Fuge gestellt, ist ein weitaus invasiverer Eingriff, wie eine periazetabuläre oder Becken-Dreifach-Osteotomie zur Verbesserung der Überdachung notwendig [37, 38].

Insgesamt gilt: Je stärker die Ausprägung der Hüftdysplasie, desto wahrscheinlicher und früher kommt es zu einer Koxarthrose [39].

Da die Hüftdysplasie primär asymptomatisch ist und das vorgeschriebene sonographische Screening erst seit Ende der 80er Jahre besteht, werden heute noch relativ häufig sog. Dysplasie-Koxarthrosen in jungem Alter diagnostiziert. Die Schädigung des Knorpels bis hin zur ausgeprägten Deformation des Hüftgelenks ist meist so weit fortgeschritten, dass nur noch ein endoprothetischer Gelenkersatz als Therapie angeboten werden kann ( $\bullet$ Abb. 8). 


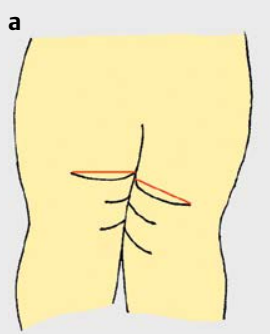

b

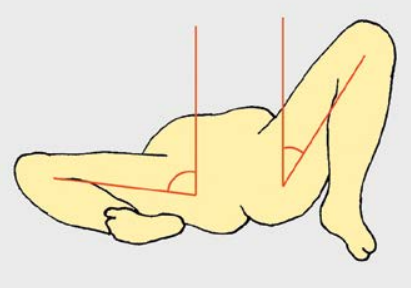

c

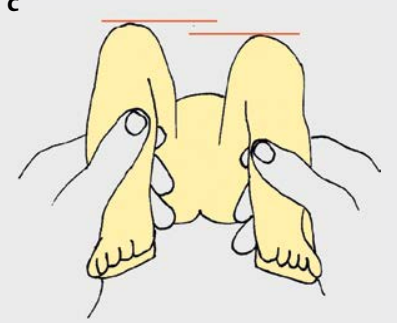

D Abb. 6 Klinische Zeichen einer linksseitigen Hüftgelenks(sub)luxation.
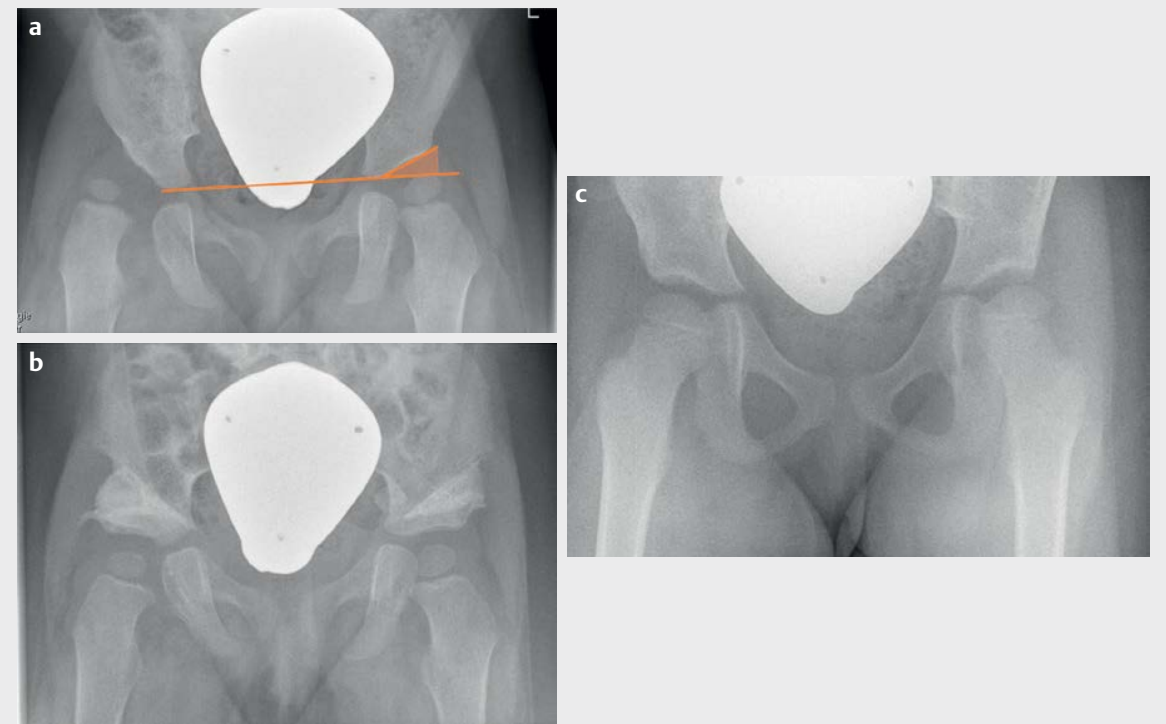

Abb. 7 a Beckenübersicht eines Patienten mit beidseitiger Hüftdysplasie bei linksseitig vergrößertem AC-Winkel und rechtsseitiger Hüftluxation, b Z. n. Acetabuloplastik bds. mit tutogenem Knochenkeil sowie rechtsseitig offen chirurgischer Einstellung, c Verlaufskontrolle 4 Jahre postoperativ: die Hüfte ist beidseits gut überdacht, der Knochenkeil vollständig konsolidiert.

Die operative Therapie von Deformitäten des proximalen Femurs ist in erster Linie eine Containment-verbessernde Umstellungsosteotomie. Dabei ist eine der am häufigsten angewandten Techniken die intertrochantäre Osteotomie mittels (Klingen-)plattenosteosynthese. Diese erfolgt je nach Deformität (de)rotierend, valgisierend oder varisierend, flektierend oder extendierend [40]. Postoperativ ist häufig eine Ruhigstellung mittels Becken-Bein-Gips und / oder eine Entlastung für 4-6 Wochen notwendig. Nach 2 Jahren kann bei abgeschlossener knöcherner Konsolidierung die Materialentfernung geplant werden.

\section{Achsfehlstellung}

Abhängig davon, in welchem Alter eine behandlungsbedürftige Achsabweichung der Beine festgestellt wird, sind verschiedene Therapieformen möglich, wobei ein rein konservativer Ansatz nicht umsetzbar ist. Generell wird die Wachstumslenkung von der Korrekturosteotomie unterschieden.
Wurde bei einem Kind mit ausreichendem Wachstumspotenzial in den kniegelenksnahen Fugen eine übermäßige Valgus- oder Varusstellung ausgemacht, die unbehandelt zu einer möglichen Arthrose führen könnte, kann das Verfahren der temporären Hemiepiphysiodese zum Einsatz kommen. Hierbei wird die entsprechend zu therapierende Wachstumsfuge mittels operativ eingebrachten Implantats auf der konvenxen Seite der Fehlstellung angehalten, sodass das Wachstum nur auf der konkaven Seite fortgesetzt werden kann. Es kann hierfür aus einer Vielzahl an Implantaten gewählt werden, von denen heute vorrangig flexible Schrauben-/Platten-Systeme oder Klammern verwendet werden. Alle drei Monate werden radiologische Verlaufskontrolle durchgeführt, mit denen man die kontinuierliche Korrektur der Fehlstellung verfolgt. Ist eine vollständige Korrektur erreicht, wird die Explantation geplant ( Abb. 9).

Bei diesem Therapieverfahren ist der gewählte Zeitpunkt der Implantation wichtig, da eine zu frühe Therapie ein Rezidiv der Kniegelenksfehlstellung (sog. Rebound-Phänomen) nach Implan- 

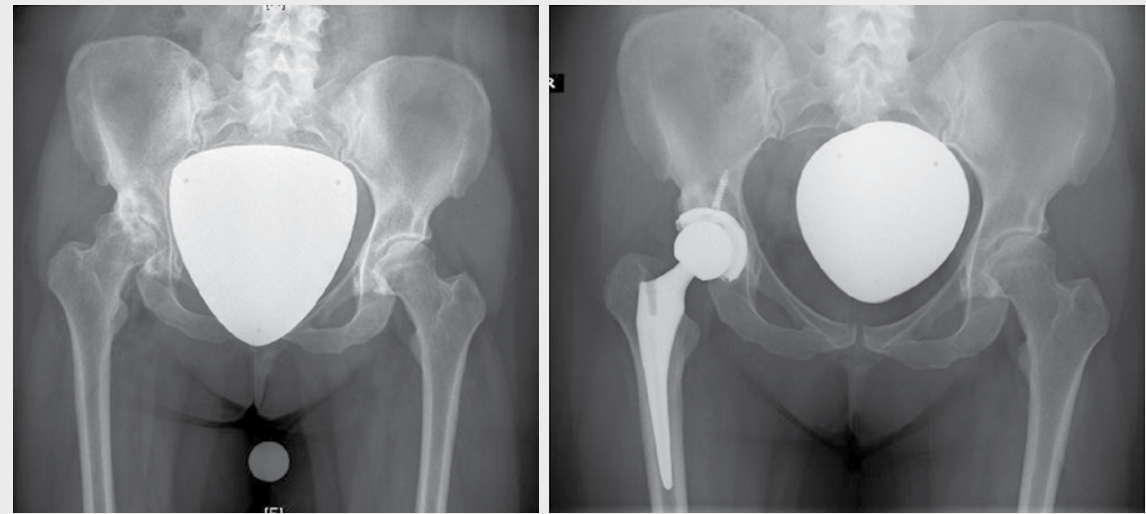

Abb. 8 Dysplasiecoxarthrose rechts bei Patientin im Alter von 42 Jahren sowie endoprothetischer Gelenkersatz.
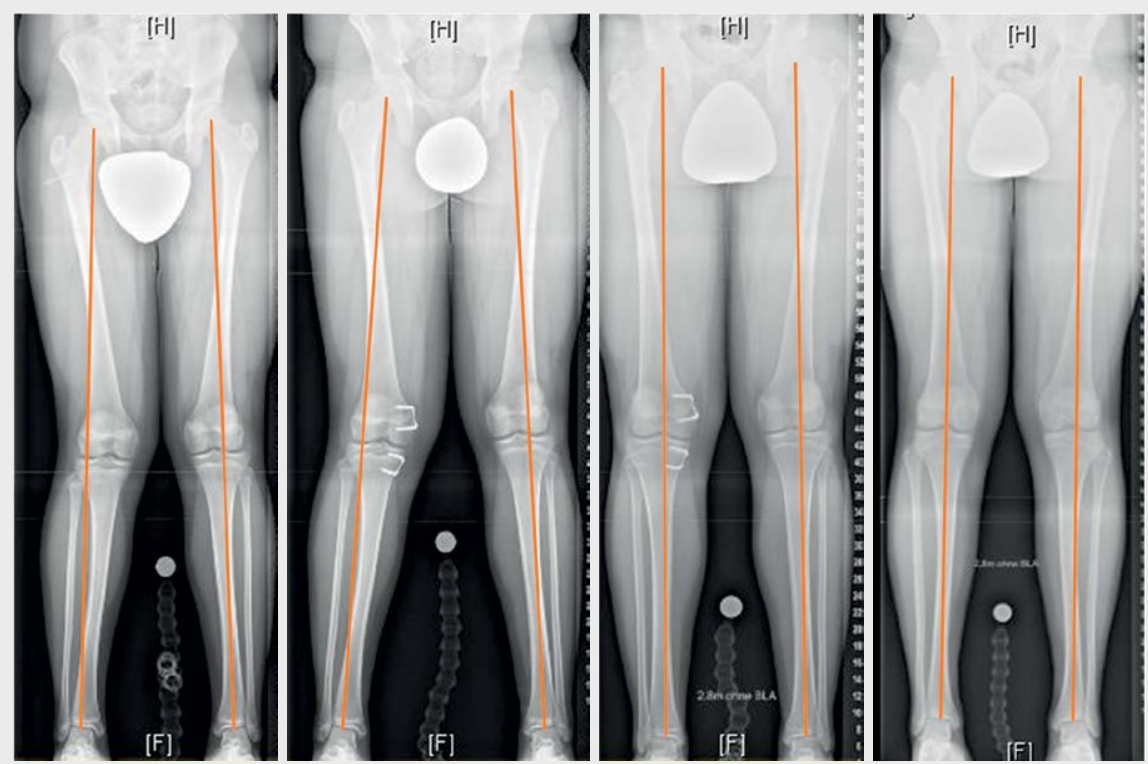

Abb. 9 Wachstumslenkung bei Genu valgum rechts mittels FlexTacks ${ }^{\top \mathrm{M}}$ : Bei Wachstumsabschluss zeigt sich rechts nach Materialentfernung eine gerade Beinachse.

tatentfernung nach sich ziehen kann. Ebenfalls kann bei zu spätem Therapiebeginn möglicherweise eine Restdeformität verbleiben, da eine vollständige Korrektur, bedingt durch zu geringes Wachstumspotenzial, nicht mehr erreicht werden kann [41].

Die Therapiedauer ist vom jeweiligen Wachstum der behandelten Fuge abhängig. Im Durchschnitt wird eine Verbesserung des mLDFW um ca. $0,7^{\circ} /$ Monat und des MPTW um ca. $0,5^{\circ}$ /Monat beobachtet [42].

Bei dieser eleganten Operationsmethode sind Komplikationen selten (3\%) und minderschwer, sodass die minimalinvasive Wachstumslenkung zur Korrektur von Achsfehlstellungen zu bevorzugen ist. Postoperativ kann umgehend die schmerzadaptierte Vollbelastung erfolgen, die durchschnittliche postoperative Verweildauer beträgt im Schnitt unter 3 Tagen.
Sobald das Skelettwachstum abgeschlossen ist, kann eine Wachstumslenkung nicht mehr durchgeführt werden. Bei ausgeprägter Fehlstellung oder einer klinischen Symptomatik ist dann eine Korrekturosteotomie anzuraten, bei der die Osteosynthese mittels Drähten, Schrauben, Platten, Marknägeln oder selten externen Fixateuren erfolgen kann. Geringer ausgeprägte gelenknahe Deformitäten werden meist mittels Plattenosteosynthese in open-wedge oder closed-wedge Technik behandelt, bei der nach Osteotomie entweder ein Aufklappen oder ein Entfernen eines Knochenkeils erfolgt. Einer der häufigsten durchgeführten Eingriffe ist die hohe Tibiaosteotomie (HTO). Hierbei wird mittels Plattenosteosynthese die neu erreichte Stellung fixiert und die knöcherne Konsolidierung abgewartet. Nach 2 Jahren kann in der Regel eine Materialentfernung stattfinden $[43,44]$. 
Zwar sind die Risiken einer kniegelenksnahen Umstellungsosteotomie ebenfalls relativ gering, jedoch gibt es deutliche Nachteile gegenüber der Wachstumslenkung, wie z. B. die erhöhte Invasivität und verlängerte Operationsdauer sowie die deutlich stärker eingeschränkte postoperative Mobilität.

Liegt eine ausgeprägte mehrdimensionale oder akut nicht korrigierbare Fehlstellung vor, kann eine komplexe Rekonstruktion mittels externen Fixateurs notwendig werden. Nach perkutaner Implantation von Pinschrauben und / oder Drähten zur Fixation der Fixateureinheiten wird in der Regel im Bereich der Deformität eine Osteotomie gesetzt. Nach einem vorgegebenen Protokoll stellt der Patient postoperativ täglich die Fixateureinheiten zueinander ein, sodass schrittweise eine graduelle Korrektur der Deformität erreicht werden kann. Nach knöcherner Konsolidierung kann die Entfernung des Fixateurs erfolgen [45].

\section{Schlussfolgerung}

Obwohl die deformitätsbedingte sekundäre Arthrose in Knie- und Hüftgelenk nur einen geringen Anteil aller diagnostizierten Gelenksarthrosen der unteren Extremitäten darstellt, sind hier die eindeutige Zuordnung der Deformität als Ursache und ein deutlich früheres Manifestationsalter der Arthrose offensichtlich. Mit Einführung der vorgeschriebenen Früherkennungsuntersuchungen U1-U9 ist die Diagnostik solcher präarthrotischen Deformitäten deutlich verbessert worden: Seit Einführung der Hüftgelenksultraschalluntersuchung in Deutschland im Jahr 1996 konnte die Anzahl der operativen Interventionen bei Hüftdysplasie und Hüftluxation um $80 \%$ reduziert werden [46].

Trotzdem bleibt eine der wichtigsten Aufgaben der Orthopädie die Früherkennung und Therapieeinleitung, denn das Versäumen des rechtzeitigen Behandlungsbeginns kann zur Erweiterung der (häufig dann auch operativen) Maßnahmen zur gelenkerhaltenden Deformitätenkorrektur oder gar zum frühzeitigen künstlichen Gelenkersatz führen. Gleichzeitig sollte nochmals erwähnt werden, dass nicht jede vermeintliche Fehlstellung auch eine präarthrotische Deformität darstellt.

Aktuell bleiben trotz intensiver Forschung noch viele Fragen der Arthroseentwicklung ungeklärt. Beispielsweise impliziert eine Varusfehlstellung am Kniegelenk mit einer höheren Wahrscheinlichkeit eine spätere Arthrose als ein Genu valgum [47]. Dennoch scheint eine Korrelation zwischen Ausprägung der Deformität, Arthrosewahrscheinlichkeit und Diagnosealter vorzuliegen [48].

Es wird somit empfohlen, bei abklärungsbedürftigen Deformitäten der unteren Extremität eine zeitnahe klinische und bedarfsweise radiologische Diagnostik zu erheben, um die bestmögliche Therapie für den Patienten zu gewährleisten.

\section{Interessenkonflikt}

Die Autorinnen/Autoren geben an, dass kein Interessenkonflikt besteht.
[1] Informationssystem der Gesundheitsberichterstattung des Bundes Häufigste Diagnosen in Praxen von Orthopädinnen und Orthopäden (in \% der Behandlungsfälle) nach Geschlecht, Rang und Anteil. Nordrhein, ab 2002

[2] Ryd L, Brittberg M, Eriksson K et al. Pre-osteoarthritis: definition and diagnosis of an elusive clinical entity. Cartilage 2015; 6: 156-165

[3] Bleß HH, Kip M., (Hrsg.). Weißbuch Gelenkersatz: Versorgungssituation endoprothetischer Hüft- und Knieoperationen in Deutschland. Springer-Verlag; 2016

[4] Günther KP, Puhl W, Brenner $\mathrm{H}$ et al. Klinische Epidemiologie von Hüft-und Kniegelenkarthrosen: Eine Übersicht über Ergebnisse der „Ulmer Osteoarthrose-Studie“. Zeitschrift für Rheumatologie 2002; 61: 244-249

[5] O'Quinn JR, Hennekam RC, Jorde LB et al. Syndromic ectrodactyly with severe limb, ectodermal, urogenital, and palatal defects maps to chromosome 19. Am J Hum Genet 1998; 62: 130-135

[6] Riddle RD, Tabin C. How limbs develop. Sci Am 1999; 280: 74-79

[7] Kraus T, Steinwender G. Kindliche Beinachsen und Füße in ihrer physiologischen Entwicklung. Pädiatrie \& Pädologie 2014; 49: 8-13

[8] Rösch C, Götz D, Haase M et al. Jahresbericht des Bundeslandes Sachsen-Anhalt zur Häufigkeit von congenitalen Fehlbildungen und Anomalien sowie genetisch bedingten Erkrankungen. Magdeburg: Fehlbildungsmonitoring Sachsen-Anhalt an der Medizinischen Fakultät der Otto-von-Guericke-Universität Magdeburg; 2004

[9] Superti-Furga A. Dysostosen. In Lentze MJSchaub JSchulte FJSpranger J, Hrsg. Pädiatrie: Grundlagen und Praxis. Springer-Verlag; 2015: 1-16

[10] Thulstrup AM, Bonde JP. Maternal occupational exposure and risk of specific birth defects. Occup Med 2006; 56: 532-543

[11] Tönnis D. Die angeborene Hüftdysplasie und Hüftluxation in Kindesund Erwachsenenalter. Grundlagen, Diagnostik, konservative und operative Behandlung. Berlin, Heidelberg, New York: Springer Verlag; 1984

[12] Graf R. Sonographie der Säuglingshüfte und therapeutische Konsequenzen: ein Kompendium. 6. Aufl. Stuttgart: Georg Thieme Verlag; 2013

[13] Shaw BA, Lee SS. Evaluation and referral for developmental dysplasia of the hip in infants. Pediatrics 2016; 138: e20163107

[14] Fraitzl CH, Kappe T, Reichel H. Das femoroacetabuläre Impingement - eine häufige Ursache des Leistensschmerzes beim Sportler. Deutsche Zeitschrift für Sportmedizin 2010; 61: 292

[15] Tretow H, Vogt B. Kniegelenk Teil I - Achsendeformitäten. Orthopädie und Unfallchirurgie up2date 2017; 12: 547-566

[16] Salenius P, Vankka E. The development of the tibiofemoral angle in children. J Bone Joint Surg Am 1975; 57: 259-261

[17] Gruskay JA, Fragomen AT, Rozbruch SR. Idiopathic rotational abnormalities of the lower extremities in children and adults. JBJS reviews 2019; 7: e3

[18] Von Lanz T. Physiologic deviations in the development of the human hip joint. Schweizerische medizinische Wochenschrift 1951; 81: 1053

[19] Shands AR Jr, Steele MK. Torsion of the femur: a follow-up report on the use of the Dunlap method for its determination. JBJS 1958; 40 : 803-816

[20] Hackenbroch M. Die Arthrosis deformans der Hüfte: Grundlagen und Behandlung. Leipzig: Thieme Verlag; 1943

[21] Leunig M, Ganz R. Femoroacetabuläres Impingement. Der Unfallchirurg 2005; 108: 9-17

[22] Paley D, Pfeil C. Prinzipien der kniegelenksnahen Deformitätenkorrektur. Orthopäde 2000; 29: 18-38 
[23] Oest O. Die Achsenfehlstellung als präarthrotische Deformität für das Kniegelenk und die röntgenologische Beinachsenbeurteilung. Unfallheilkunde 1978; 81: 629-633

[24] Bruns J, Steinhagen J. Der Knorpelschaden als präarthrotische Deformität - Biologische Grundlagen. Deutsche Zeitschrift für Sportmedizin 2000; 51: 42-47

[25] Dt. Ges. f. Orthopädie und orthopäd. Chirurgie + BV d. Ärzte f. Orthopädie, (Hrsg.). Leitlinien der Orthopädie. 2. Auflage Köln: Dt: Ärzte-Verlag; 2002

[26] Casser HR, Sonographische Untersuchung der Säulingshüfte.In Hofmann V. Ultraschalldiagnostik in Pädiatrie und Kinderchirurgie. 3. Auflage Stuttgart: Georg Thieme Verlag; 2005

[27] Paley D. Multiplier method for predicting limb-length discrepancy. JBJS 2000; 82: 1432-1446

[28] Strecker W. Osteotomien - Hüftnaher Femur. OP-Journal 2011; 27 : 240-247

[29] Wirtz C, Wanner R, Kohl S et al. Knie-Achsendeviationen - Ursache für Gonarthrose? Schweizerische Zeitschrift für Sportmedizin und Sporttraumatologie 2011; 59: 169-173

[30] Westhoff B, Jäger M, Krauspe R. Kindliche Beinachsen - Was ist pathologisch? Orthopäde 2007; 36: 485

[31] Greulich W, Pyle SL. Radiographic atlas of skeletal development of the hand and wrist. 2. Auflage Stanford: Stanford University Press; 1959

[32] Schrading S, Schulze A. Präoperative bildgebende Diagnostik und Planung. Orthopäde 2016; 45: 644

[33] Argentieri E, Burge A. Magnetic Resonance Imaging of Articular Cartilage within the Knee. J Knee Surg 2018; 31: 155-165

[34] Eagle S, Potter H, Koff M. Morphologic and quantitative magnetic resonance imaging of knee articular cartilage for the assessment of post-traumatic osteoarthritis. J Orthop Res 2017; 35: 412-423

[35] Matthiessen HD, Wachstum, Reifung und Dynamik im Säuglingshüftpfannendach - Experimentelle Untersuchungen an Wachstumsfugen. In: Konermann W, Gruber G, Tschauner C., Hrsg. Die Hüftreifungsstörung. Heidelberg: Steinkopff; 1999

[36] Gulati V, Eseonu K, Sayani J et al. Developmental dysplasia of the hip in the newborn: A systematic review. World J Orthop 2013; 4: 32-41

[37] Kaneuji A, Sugimori T, Ichiseki T et al. Rotational acetabular osteotomy for osteoarthritis with acetabular dysplasia: conversion rate to total hip arthroplasty within twenty years and osteoarthritis progression after a minimum of twenty years. JBJS 2015; 97: 726-773
[38] Tönnis D, Pfannendacheingriffe zur Beseitigung der Hüftdysplasie. In: Tönnis D. Die angeborene Hüftdysplasie und Hüftluxation im Kindes-und Erwachsenenalter: Grundlagen, Diagnostik, konservative und operative Behandlung. Berlin, Heidelberg: Springer-Verlag; 2013

[39] Jacobsen S, Sonne-Holm S, Søballe K et al. Hip dysplasia and osteoarthrosis: a survey of 4151 subjects from the Osteoarthrosis Substudy of the Copenhagen City heart study. Acta orthopaedica 2005; 76: 149-158

[40] Schai P, Exner GU. Die intertrochantäre Korrekturosteotomie nach Imhäuser. Operative Orthopädie und Traumatologie 2007; 19: 368-388

[41] Vogt B, Schiedel F, Rödl R. Wachstumslenkung bei Kindern und Jugendlichen. Der Orthopäde 2014; 43: 267-284

[42] Hagedorn T. Wachstumslenkung bei Beinlängendifferenzen und Beinachsenfehlstellungen. Klinische und radiologische Evaluation der temporären Epiphysiodese mittels Orthofix eight-Plate [Dissertation], Münster: Westfälische Wilhelms Universität Münster. 2013

[43] Wu L, Lin J, Jin Z et al. Comparison of clinical and radiological outcomes between opening-wedge and closing-wedge high tibial osteotomy: A comprehensive meta-analysis. PLoS ONE 2017; 12: e0171700

[44] Galla M, Lobenhoffer P. Die öffnende valgisierende Umstellungsosteomie der proximalen Tibia mit dem TomoFix ${ }^{\mathrm{TM}}$-Plattenfixateur. Operative Orthopädie und Traumatologie 2004; 16: 397

[45] Lichte P, Kobbe P, Lörken M et al. Planung von Korrekturosteotomien der unteren Extremität. Unfallchirurg 2010; 113: 573

[46] Baumgart K, Mellerowicz H. Hüftdysplasie. Orthopädie und Unfallchirurgie up2date 2013; 8: 3-22

[47] Brouwer GM, Tol AV, Bergink AP et al. Association between valgus and varus alignment and the development and progression of radiographic osteoarthritis of the knee. Arthritis \& rheumatism 2007; 56 : 1204-1211

[48] Frank W, Oest O, Rettig H. Die Röntgenganzaufnahme in der Operationsplanung von Korrekturosteotomien der Beine. Z Orthop 1974; 112: 344-347 